\title{
值得玩味的几道竞赛题
}

\author{
徐汪华 ${ }^{*}$ \\ 安庆师范大学化学化工学院, 安徽 安庆 246011
}

摘要: 借助对几道化学竞赛题进行解析, 提高学生的空间想象能力和发散思维能力, 同时提出了有益的建议。

关键词: 化学竞赛; 正四面体烷; 环丁二烯；立方烷；环辛四烯；空间想象力；发散思维

中图分类号: G64； O6; G633.8

\section{Analysis on Several Valuable Chemical Contest Problems}

\section{Wanghua $\mathrm{Xu}$ *}

School of Chemistry and Chemical Engineering, Anqing Normal University, Anqing 246011, Anhui Province, P. R. China.

Abstract: Through the analysis of several valuable chemical contest problems, the students' spatial visualization and divergent thinking abilities were improved, and the teaching's advice was provided.

Key Words: Chemistry contest; Tetra-tert-butyltetrahedrane; 1,3-Cyclobutadiene; Cubane; Cyclooctatetraene; Spatial visualization; Divergent thinking

怎样开发学生的智力, 提高学生的能力, 这是一个永远值得探讨的课题。有些优秀的竞赛题不 仅考查学生的空间想象力, 而且考查学生的发散思维能力, 其答案往往不是唯一的。下面结合本人 的观点对几道化学竞赛题进行解析, 希望能帮助学生提高空间想象能力和发散思维能力, 也能启迪 教师运用新方法、新思想来进行教学, 从而提高教学效率。

\section{1 试题解析}

例 1 有人设想某种分子式为 $\mathrm{C}_{4} \mathrm{~N}_{4} \mathrm{O}_{8}$ 的物质(该物质中同种原子的化学环境完全相同)是一种威 力极强的炸药, 请推测它的结构简式。

[答案]

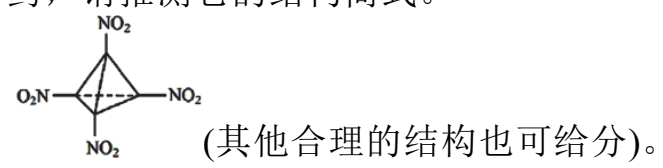

[解析] 这是 2003 年浙江省化学竞赛试题第 22 题的第 4 小题, 主要考查学生的空间想象能力和 逻辑推理能力。

计算有机物的不饱和度 $\Omega$ 可以预测其结构。若某有机物的化学式为 $\mathrm{C}_{x} \mathrm{H}_{y} \mathrm{X}_{z} \mathrm{R}_{w} \mathrm{M}_{p}$, 其中 $\mathrm{X}$ 代表 $\mathrm{F} 、 \mathrm{Cl} 、 \mathrm{Br}$ 等, $\mathrm{R}$ 代表 $\mathrm{O} 、 \mathrm{~S}$ 等, $\mathrm{M}$ 代表 $\mathrm{N} 、 \mathrm{P}$ 等, 则 $\Omega=(2 x+2-y-z+p) / 2, w$ 不纳入计算。若 $\Omega=1$, 则分子中可能含有 1 个双键如: $\mathrm{C}=\mathrm{C}$ 或 $\mathrm{C}=\mathrm{O}$ 或 $\mathrm{C}=\mathrm{N}$ 或 $\mathrm{N}=\mathrm{N}$ 或 $\mathrm{C}=\mathrm{S}$ 或 1 个单环等。若 
$\Omega=2$, 则分子中可能含有 1 个三键如: $\mathrm{C} \equiv \mathrm{C}$ 或 $\mathrm{C} \equiv \mathrm{N}$ 或 $\mathrm{N} \equiv \mathrm{N}$ 或 2 个双键或 2 个单环或 1 个双键和 1 个单环等。依此类推。若 $\Omega \geq 4$ 且 $x \geq 6$, 分子中可能含有苯环。不管怎样, $\Omega=$ 双键数 + 三键数 $\times$ $2+$ 环数, 如: $\|, \Omega=2+0 \times 2+1=3$ 。但对立体封闭有机物分子(多面体或笼状结构)来说, $\Omega=$ 双键数 + 三键数 $\times 2+$ 环数 -1 。如: $A, \Omega=0+0 \times 2+4-1=3$ 。

$\mathrm{C}_{4} \mathrm{~N}_{4} \mathrm{O}_{8}$ 的不饱和度 $\Omega=(2 \times 4+2+4) / 2=7$, 且 $n(\mathrm{~N}): n(\mathrm{O})=1: 2$, 结合它 “是一种威力极强的 炸药” 的信息, 推测该分子中可能含有 4 个 $-\mathrm{NO}_{2}$ (共有 4 个不饱和度, 每个 $-\mathrm{NO}_{2}$ 的不饱和度为 1 ) 且键的张力大, 其余 4 个 $\mathrm{C}$ 贡献 3 个不饱和度。在不考虑画出的结构式是否确实存在或稳定的前提 下，进一步推测该分子可能的结构如图 1 所示。再结合 “该物质中同种原子的化学环境完全相同” 的信息，最终推测该分子可能的结构为图 $1 \mathrm{~g}$ 或图 $1 \mathrm{~h}$ ，而图 $1 \mathrm{a}-\mathrm{f}$ 不符合题意。

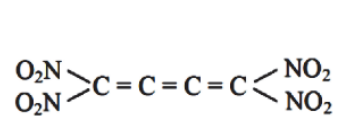

(a)

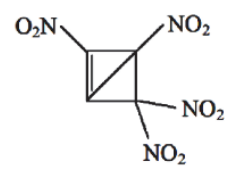

(e)

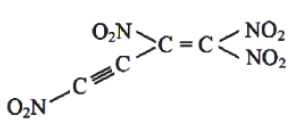

(b)

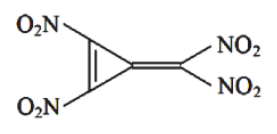

(c)

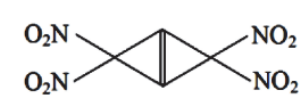

(d)

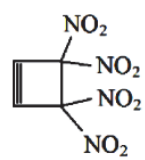

(f)<smiles>O=[N+]([O-])C1C([N+](=O)[O-])C([N+](=O)[O-])C1[N+](=O)[O-]</smiles>

(g)

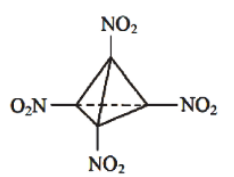

(h)

图 $1 \mathrm{C}_{4} \mathrm{~N}_{4} \mathrm{O}_{8}$ 的可能结构式示意图

例 2 最近, 我国一留美化学家参与合成了一种新型炸药, 它跟三硝基甘油一样抗打击、抗震, 但一经引爆就会发生激烈爆炸, 据信是迄今最烈性的非核爆炸品。该炸药的化学式为 $\mathrm{C}_{8} \mathrm{~N}_{8} \mathrm{O}_{16}$, 同种 元素的原子在分子中是毫无区别的。

（1）把它的结构式画在下面方框内。

(2) 把它的爆炸反应方程式写在下面的方框内。

（3）它具有强烈爆炸性的原因是: (注：只需给出要点即可)

[答案] (1)

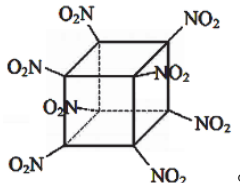

答八硝基环辛四烯者也按正确论。

(2) $\mathrm{C}_{8}\left(\mathrm{NO}_{2}\right)_{8}=8 \mathrm{CO}_{2} \uparrow+4 \mathrm{~N}_{2} \uparrow$ 。

(3) (1) 八硝基立方烷分解得到的两种气体都是最稳定的化合物。(2) 立方烷的碳架键角 只有 $90^{\circ}$, 大大小于 $109^{\circ} 28^{\prime}$ (或答：是张力很大的环), 因而八硝基立方烷是一种高能化合物, 分解 时将释放大量能量。(3) 八硝基立方烷分解产物完全是气体, 体积膨胀将引起激烈爆炸。(只要能答 出类似以上要点者均可得满分)

[解析] 这是 2000 年全国化学竞赛初赛试题第 9 题, 主要考查学生的空间想象能力和逻辑推理 能力。

$\mathrm{C}_{8} \mathrm{~N}_{8} \mathrm{O}_{16}$ 的不饱和度 $\Omega=(2 \times 8+2+8) / 2=13$, 且 $n(\mathrm{~N}): n(\mathrm{O})=1: 2$, 结合它是 “一种新型炸 药, 它跟三硝基甘油一样抗打击、抗震, 但一经引爆就发生激烈爆炸, 据信是迄今最烈性的非核爆 炸品” 的信息, 推测该分子中可能含有 8 个 $-\mathrm{NO}_{2}$ (共有 8 个不饱和度)且键的张力大, 其余 8 个 $\mathrm{C}$ 贡献 5 个不饱和度。在不考虑画出的结构式是否确实存在或稳定的前提下，进一步推测该分子可能 的结构如图 2 所示。再结合 “同种元素的原子在分子中是毫无区别的” 的信息, 最终推测该分子可 能的结构为图 $2 \mathrm{~g}$, 而图 $2 \mathrm{a}-\mathrm{f}$ 不符合题意。 


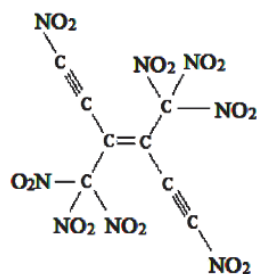

(a)

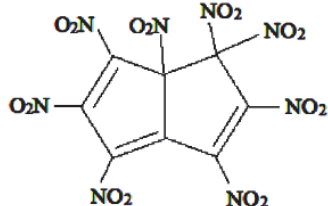

(b)<smiles>O=[N+]([O-])C(=C([N+](=O)[O-])[N+](=O)[O-])c1c([N+](=O)[O-])c([N+](=O)[O-])c([N+](=O)[O-])c([N+](=O)[O-])c1[N+](=O)[O-]</smiles>

(c)

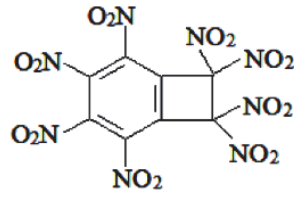

(d)<smiles>O=C([N+](=O)[O-])C1([N+](=O)[O-])C([N+](=O)[O-])([N+](=O)[O-])C([N+](=O)[O-])=C([N+](=O)[O-])C1([N+](=O)[O-])[N+](=O)[O-]</smiles>

(e)<smiles>O=[N+]([O-])C1=C([N+](=O)[O-])C([N+](=O)[O-])=C([N+](=O)[O-])C([N+](=O)[O-])C([N+](=O)[O-])=C1[N+](=O)[O-]</smiles>

(f)

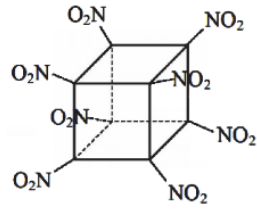

(g)

图 $2 \mathrm{C}_{8} \mathrm{~N}_{8} \mathrm{O}_{16}$ 的可能结构式示意图

例 $3 \mathrm{~A} 、 \mathrm{~B} 、 \mathrm{C} 、 \mathrm{D}$ 四种烃, 摩尔质量之比是 $1: 2: 3: 4$, 且它们的实验式都是 $\mathrm{C}_{7} \mathrm{H}_{5}$, 都只有 3 种一硝基取代物。写出 $\mathrm{A} 、 \mathrm{~B} 、 \mathrm{C} 、 \mathrm{D}$ 的结构简式(各写一例):

[答案]
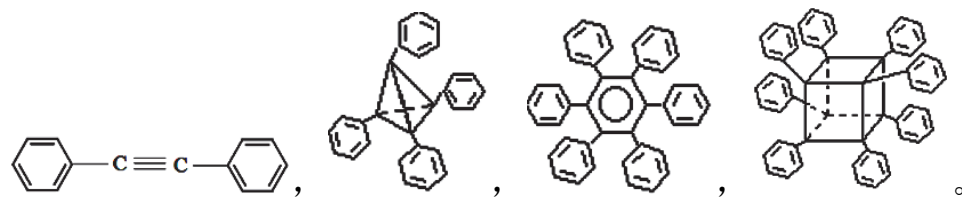

[解析] 这是 2005 年浙江省化学竞赛试题第 25 题的第 2 小题, 主要考查学生的空间想象能力和 逻辑推理能力。

根据 “它们的实验式都是 $\mathrm{C}_{7} \mathrm{H}_{5}$ ” 可设 A、B、C、D 四种烃的化学式分别为 $\left(\mathrm{C}_{7} \mathrm{H}_{5}\right)_{p} 、\left(\mathrm{C}_{7} \mathrm{H}_{5}\right)_{q}$ 、 $\left(\mathrm{C}_{7} \mathrm{H}_{5}\right)_{r}$ 、 $\left(\mathrm{C}_{7} \mathrm{H}_{5}\right)_{\mathrm{s}}$ 。再根据 “摩尔质量之比是 $1: 2: 3: 4$ ” 可推知 $89 p: 89 q: 89 r: 89 s=1: 2: 3: 4 \Rightarrow$ $p: q: r: s=1: 2: 3: 4$ 。由烃的通式 $\left[\mathrm{C}_{n} \mathrm{H}_{2 n+2}\right.$ (链状烷烃)、 $\mathrm{C}_{n} \mathrm{H}_{2 n}$ (链状单烯烃或单环烷烃)、 $\mathrm{C}_{n} \mathrm{H}_{2 n-2}$ (链状单炔烃或链状二烯烃或单环单烯烃)、 $\mathrm{C}_{4 n+2} \mathrm{H}_{2 n+4}$ (苯、奈、葱等稠环芳香烃)、……可知: 烃的 化学式中 $\mathrm{H}$ 原子数为偶数 $\Rightarrow p 、 q 、 r 、 s$ 应为偶数 $2 、 4 、 6 、 8$ 等。

当 $p=2$ 时, $\mathrm{A}$ 的化学式为 $\left(\mathrm{C}_{7} \mathrm{H}_{5}\right)_{2}$ 即 $\mathrm{C}_{14} \mathrm{H}_{10}$, 其 $\Omega=(2 \times 14+2-10) / 2=10$, 推测该分子中可 能含有 2 个苯环(共有 8 个不饱和度, 每个苯环 $-\mathrm{C}_{6} \mathrm{H}_{5}$ 的不饱和度为 4 ), 其余 2 个 $\mathrm{C}$ 贡献 2 个不饱 和度, 可能为 $\mathrm{C} \equiv \mathrm{C}$ 。在不考虑画出的结构式是否确实存在或稳定的前提下, 进一步推测该分子可能 的结构为:

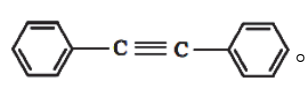
该结构的一硝基取代物只有:
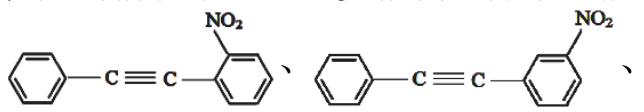
《 $\mathrm{C} \equiv \mathrm{c}-\mathrm{NO}_{2}$ 等 3 种, 符合题意。

当 $q=4$ 时, $\mathrm{B}$ 的化学式为 $\left(\mathrm{C}_{7} \mathrm{H}_{5}\right)_{4}$ 即 $\mathrm{C}_{28} \mathrm{H}_{20}$, 其 $\Omega=(2 \times 28+2-20) / 2=19$, 推测该分子中可能 含有 4 个苯环(共有 16 个不饱和度), 其余 4 个 $\mathrm{C}$ 贡献 3 个不饱和度。在不考虑画出的结构式是否确 实存在或稳定的前提下, 进一步推测该分子可能的结构如图 3 所示(其中 $\mathrm{Ph}$ 代表苯环)。其中符合题 意的一硝基取代物只有 3 种的为图 3a、3d、3f、3g、3h 等，而图 3b、3c、3e 等不符合题意。

当 $r=6$ 时, $\mathrm{C}$ 的化学式为 $\left(\mathrm{C}_{7} \mathrm{H}_{5}\right)_{6}$ 即 $\mathrm{C}_{42} \mathrm{H}_{30}$, 其 $\Omega=(2 \times 42+2-30) / 2=28$, 推测该分子中可能 含有 6 个苯环(共有 24 个不饱和度), 其余 6 个 C 贡献 4 个不饱和度。在不考虑画出的结构式是否确 实存在或稳定的前提下, 进一步推测该分子可能的结构如图 4 所示。其中符合题意的一硝基取代物 只有 3 种的为图 $4 \mathrm{a} 、 4 \mathrm{~h} 、 4 \mathrm{i}$ 等, 而图 $4 \mathrm{~b}-\mathrm{g}$ 不符合题意。 
<smiles>C(=Cc1ccccc1)=C(c1ccccc1)c1ccccc1</smiles>

(a)

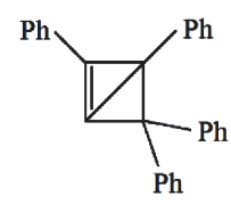

(e)<smiles>C(#Cc1ccccc1)C=C(c1ccccc1)c1ccccc1</smiles>

(b)<smiles>c1ccc(C2=C(c3ccccc3)C2c2ccccc2)cc1</smiles>

(c)

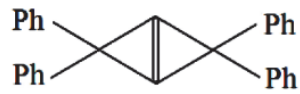

(d)

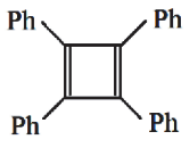

(g)

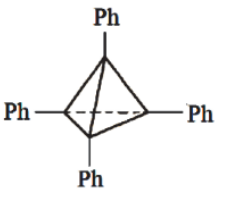

(h)

图 $3\left(\mathrm{C}_{7} \mathrm{H}_{5}\right)_{4}$ 的可能结构式示意图<smiles>C(#CC(c1ccccc1)(c1ccccc1)c1ccccc1)C=C(c1ccccc1)c1ccccc1</smiles>

(a)<smiles>C(=C/C1c2ccccc2-c2ccccc21)\c1ccccc1</smiles>

(b)

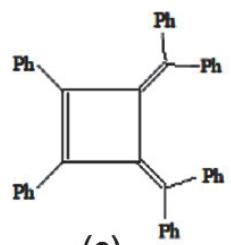

(c)

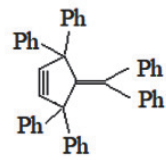

(d)<smiles></smiles>

(e)<smiles></smiles>

(f)<smiles>c1ccc(C2=C(c3ccccc3)C3(c4ccccc4)C(c4ccccc4)C2C3c2ccccc2)cc1</smiles>

(g)

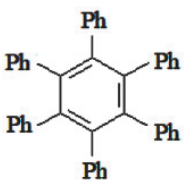

(h)

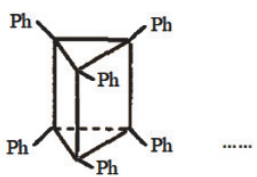

(i)

图 $4\left(\mathrm{C}_{7} \mathrm{H}_{5}\right)_{6}$ 的可能结构式示意图

当 $s=8$ 时, $\mathrm{D}$ 的化学式为 $\left(\mathrm{C}_{7} \mathrm{H}_{5}\right)_{8}$ 即 $\mathrm{C}_{56} \mathrm{H}_{40}$, 其 $\Omega=(2 \times 56+2-40) / 2=37$, 推测该分子中可能 含有 8 个苯环(共有 32 个不饱和度), 其余 8 个 $\mathrm{C}$ 贡献 5 个不饱和度。在不考虑画出的结构式是否确 实存在或稳定的前提下, 进一步推测该分子可能的结构如图 5 所示。其中符合题意的一硝基取代物 只有 3 种的为图 $5 \mathrm{~g}$, 而图 $5 \mathrm{a}-\mathrm{f}$ 不符合题意。

由此可见，这三道竞赛题题干文字不多，但给我们留下思考的余地多，回味无穷。

\section{2 与原答案的商榷}

例 2 是 2000 年全国化学竞赛初赛试题第 9 题，原答案说“答八硝基环辛四烯者也按正确论”。 笔者认为：原答案可以说 “答八硝基环辛四烯者也可以适当给分”，但不能说 “按正确论”。

环辛四烯双负离子 $\left[\mathrm{C}_{8} \mathrm{H}_{8}\right]^{2-}$ 有 10 个 $\pi$ 电子, 符合休克尔 $(4 n+2)(n=2)$ 规则, 具有芳香性, 为平 面构型的对称环多烯 ${ }^{[1]}$ 结构(图 6a)。但 $\mathrm{X}$ 射线衍射测定结果表明, 环辛四烯 $\mathrm{C}_{8} \mathrm{H}_{8}$ 分子中碳原子不在 同一平面上 ${ }^{[2]}$, 分子并不成一平面, 而成一个澡盆的形状 ${ }^{[3]}$ (图 6b)所示。也就是说, 环辛四烯 $\mathrm{C}_{8} \mathrm{H}_{8}$ 分子与环辛四烯双负离子 $\left[\mathrm{C}_{8} \mathrm{H}_{8}\right]^{2-}$ 不一样，两者的结构不同。

由图 $6 \mathrm{~b}$ 可知, 环辛四烯分子中 8 个 $\mathrm{C}$ 可分为 2 组: 盆底的 4 个 $\mathrm{C}$ (等效)为一组, 盆边的 4 个 $\mathrm{C}$ (等效)为另一组。这 2 组 $\mathrm{C}$ 所处的化学环境不同, 这 2 组 $\mathrm{C}$ 所连接的 $-\mathrm{NO}_{2}$ 的化学环境也不同(图 $6 \mathrm{c}$ )。题意要求 “同种元素的原子在分子中是毫无区别的”, 所以, 八硝基环辛四烯不符合题意, 不能 “按正确论”。严格地说, 在解答例 2 时 “答八硝基环辛四烯者” 就不对, 不应给分。但命题人心慈 手软, 考虑到能回答出这个答案的学生 “空间想象能力和发散思维能力” 是不错的, 给予鼓励, “答 八硝基环辛四烯者”也可以适当给分。 


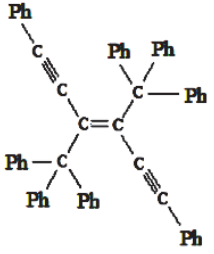

(a)<smiles>c1ccccc1</smiles>

(b)

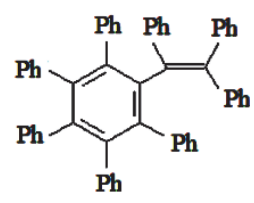

(c)

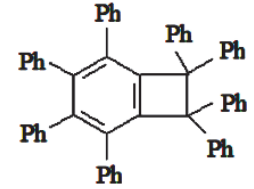

(d)

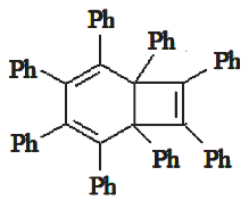

(e)

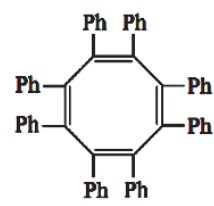

(f)

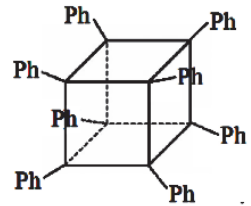

(g)

图 $5\left(\mathrm{C}_{7} \mathrm{H}_{5}\right)_{8}$ 的可能结构式示意图

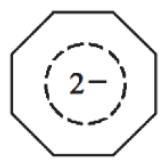

(a)

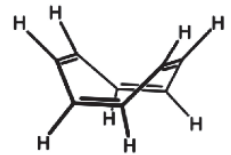

(b)

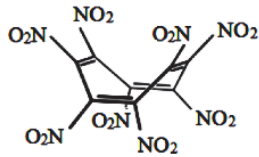

(c)

图 6 环辛四烯及其衍生物的分子结构示意图

\section{3 两点建议}

（1）注意思维定势的影响。

思维定势, 也称 “惯性思维”, 是由先前的活动而造成的一种对活动的特殊心理准备状态, 或活 动的倾向性。思维定势对问题解决既有积极的一面, 也有消极的一面, 它容易使我们养成一种呆板、 机械、千篇一律的解题习惯。当新旧问题形似质异时, 思维的定势往往会使解题者步入误区。例如:

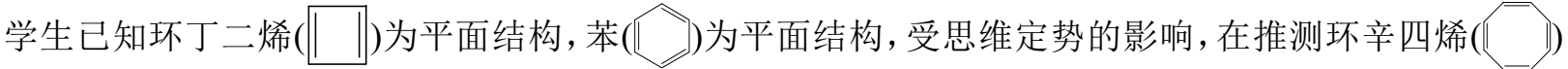
的结构时, 往往误认为其也是平面结构。当然, 我们要启发学生: 环辛四烯双负离子((2-))为平面结构。

(2) 注意思维的发散性。

发散思维, 又称辐射思维、放射思维、扩散思维或求异思维, 是指大脑在思维时呈现的一种扩 散状态的思维模式, 它表现为思维视野广阔, 思维呈现出多维发散状。例如: 在教学中, 我们把 例 1 、例 2 和例 3 等放在一起来操练, 这本身就是在培养学生的发散思维。我们在平时解析例 1 时, 在不考虑画出的结构式是否确实存在或稳定的前提下, 不仅让学生知道该分子可能的结构为图 $1 \mathrm{~h}$, 而且让学生知道图 $1 \mathrm{~g}$ 也对, 还应让学生知道图 1a-f 不符合题意。同时, 我们还应让学生比较: 为 什么例 3 中图 $3 \mathrm{a} 、 3 \mathrm{~d} 、 3 \mathrm{f} 、 3 \mathrm{~g}$ 和 $3 \mathrm{~h}$ 等符合题意，而例 1 中却只有图 $1 \mathrm{~g}$ 和 $1 \mathrm{~h}$ 符合题意?

总之, 在思考问题时, 我们既要注意思维定势的影响, 又要注意思维的发散性。只有这样, 我 们才能跳出点、线、面的限制, 进行立体式思维, 我们才能培养学生的创造力。

[1] 周公度, 段连运. 结构化学基础. 第 3 版. 北京: 北京大学出版社, 2002: 199.

[2] 王积涛, 王永梅, 张宝申, 胡青眉, 庞美丽. 有机化学(上册). 第 3 版. 天津: 南开大学出版社, 2012: 57, 185.

[3] 邢其毅, 徐瑞秋, 周政. 基础有机化学(上册). 北京: 高等教育出版社, 1987: 215. 